\title{
Insufficient fumarase contributes to generating reactive oxygen species in Dahl salt
}

\section{sensitive rats}

Entai Hou ${ }^{1 \#}$, Xuewei Zheng ${ }^{1 \#}$, Zhe Yang $^{1}$, Xian Li ${ }^{1}$, Zerong Liu ${ }^{1}$, Meng Chen ${ }^{1}$, Xiaoxue

$$
\mathrm{Li}^{1}, \text { Mingyu Liang }{ }^{2} \text {, Zhongmin } \operatorname{Tian}^{1} *
$$

${ }^{1}$ The Key Laboratory of Biomedical Information Engineering of Ministry of Education,

School of Life Science and Technology, Xi'an Jiaotong University, Xi'an 710049, China

${ }^{2}$ Center of Systems Molecular Medicine, Department of Physiology, Medical College of Wisconsin, Milwaukee, WI 53226

"These authors contributed equally to this work

*Corresponding author:

Dr. Zhongmin Tian,

School of Life Science and Technology, Xi'an Jiaotong University,

Xi'an 710049, China

Email: zmtian@xjtu.edu.cn

Phone: 86-29-82667331

Fax: 86-29-82668668 


\section{Abstract}

Dahl SS rats exhibit greater levels of renal medullary oxidative stress and lower levels of fumarase activities. Fumarase insufficiencies can increase reactive oxygen species (ROS), the mechanism of which, however, is not clear. A proteomic analysis indicated fumarase knockdown in HK-2 cells resulted in changes in the expression or activity of NADPH oxidase, mitochondrial respiratory chain complex I and III, ATP synthase subunits, and $\alpha$-oxoglutarate dehydrogenase, all of which are sites of ROS formation. Meantime, the activities of key antioxidant enzymes such as G6PD, 6PGD, GR, GPx and GST increased significantly too. The apparent activation of antioxidant defense appeared insufficient as glutathione precursors, glutathione and GSH/GSSG ratio were decreased. SS rats exhibited changes in redox metabolism similar to HK-2 cells with fumarase knockdown. Supplementation with fumarate and malate, the substrate and product of fumarase, increased and decreased, respectively, blood pressure and the levels of $\mathrm{H}_{2} \mathrm{O}_{2}$ and MDA in kidney tissues of SS rats. These results indicate fumarase insufficiencies cause a wide range of changes at several sites of ROS production and antioxidant mechanisms.

Key words: Dahl salt sensitive rats, Fumarase, Fumarate, Reactive oxygen species, Glutathione metabolism 
1

2

3

4 5 hypertension, was widely used to dissect the pathogenesis. SS. $13^{\mathrm{BN}}$ rats as a

6

\section{Introduction}

Salt-sensitive hypertension, a multifactorial disease affecting nearly $15 \%$ of the world population, is a major risk factor for stroke, heart failure, and end-stage renal disease [1-3]. Dahl salt sensitive (SS) rat, as an animal model of human salt sensitive normotensive control, which chromosome 13 of the Brown Norway (BN) rat was integrated into the genetic background of the SS and result in a significant reduction of blood pressure and renal injury [4], had tremendously narrowed the interested gene on chromosome 13 . There is only a $1.95 \%$ allelic difference over the entire genome between SS and SS.13 ${ }^{\mathrm{BN}}$ rats. Meanwhile, SS rats renal medullary exhibited obviously oxidative stress and blood pressure salt-sensitivity compared with SS. $13^{\mathrm{BN}}$ rats [5], as $\mathrm{H}_{2} \mathrm{O}_{2}$ concentrations had also been found to be nearly twice as great in the dialysate of SS rats when both rats were maintained on a $0.4 \% \mathrm{NaCl}$ diet [6]. The level was more than doubled with a $4 \% \mathrm{NaCl}$ in diet, but it remained significantly higher in SS rats [6]. Furthermore, the increased production of $\mathrm{O}_{2}{ }^{-}$in MTAL also could diffuses to surrounding vasa recta, contribute to decrease bioavailability of NO and reduce medullary blood flow, resulting in sustained hypertension in SS rats [7]. However, the mechanisms that excessive $\mathrm{O}_{2} \cdot{ }^{-}$and $\mathrm{H}_{2} \mathrm{O}_{2}$ produced in renal medulla of SS rats, is yet not fully understood.

Our previous differential proteomics study found that fumarase (FH), the gene of which locates on rat chromosome 13 , was expressed differentially between SS and $\mathrm{SS} .13^{\mathrm{BN}}$ rats, and the activity of $\mathrm{FH}$ is significantly lower in SS rats $[8,9]$. As $\mathrm{FH}$ 
catalyzes the conversion between fumarate and L-malate, the insufficiency of FH activity in SS rat kidney was associated with increased levels of fumarate [8]. The intravenous infusion of a fumarate precursor diethyl-fumarate in $\mathrm{SS} .13^{\mathrm{BN}}$ rats resulted in increased levels of fumarate in renal medullary and significantly exacerbated salt-induced hypertension in $\mathrm{SS} 13^{\mathrm{BN}}$ rats [8]. Furthermore, excessive fumarate could also increase the level of $\mathrm{H}_{2} \mathrm{O}_{2}$ in vivo and in cultured $\mathrm{HK}-2$ cells [8].

Our recent research work further demonstrated that overexpressing fumarase on the background of the SS rat, SS-TgFh1 transgenic rats, could significantly attenuate hypertension and $\mathrm{H}_{2} \mathrm{O}_{2}$ production [10]. So the present study will further dissect the imbalance of redox metabolism and understand the mechanism of ROS production by a proteomic analysis in fumarase knockdown HK-2 cells.

\section{Results}

\section{Differentially expressed proteins between FH insufficient HK-2 cells and NC cells}

The fumarase was knocked down by siRNA in HK-2 cell line, the protein profile of the whole cells was analyzed by iTRAQ based proteomics technology, and metabolites were detected by GC/MS. Content and activity of FH were examined to ensure the efficiency of FH knockdown. The sample preparation used for proteomic analysis same as the description we reported [11] and the sample test was showed in Figure EV1.

A total of 3174 distinct proteins were identified and quantified reliably at the value of global FDR less than $1 \%$ and identified with more than two peptides for $95 \%$ 
confidence. Details of the quantified proteins are shown in Table EV1. Compared with the NC, 267 identified proteins exhibited significant difference between FH knockdown HK-2 cells and NC cells (fold change $>1.5, \mathrm{P}<0.05$ ) (Table EV2). Among them, 165 proteins displayed increased expression and 102 proteins displayed decreased expression compared to NC. SOD2, IDH1, IDH2 and HSP90 among the 267 differentially expressed proteins were further confirmed by Western blotting. As shown in Figure EV2, the relative protein content from western blotting was consistent with proteomic quantification.

\section{GO and KEGG analysis of the differential expressed proteins}

To clarify the changed metabolic pathways that involved in the response to $\mathrm{FH}$ knockdown, 267 differential proteins were further examined using the Omics Bean bioinformatics tool. Gene ontology (GO) enrichment analysis was conducted in biological process (BP), cellular component (CC) and molecular function (MF) categories, respectively. An overview of significantly changed proteins in $\mathrm{BP}, \mathrm{CC}$ and MF categories was shown in Figure EV3 and Table EV3. KEGG pathway-based analysis showed that ribosome, carbon metabolism, glutathione metabolism, mRNA transport, pyruvate metabolism, fatty acid degradation, glycolysis /gluconeogenesis and TCA cycle were significantly enriched among 267 differential proteins (Figure EV4 and Table EV4).

The formation sites of excessive amounts of ROS and $\mathrm{H}_{2} \mathrm{O}_{2}$ in $\mathrm{FH}$ knockdown HK-2 cells 
insufficient HK-2 cells compared with NC cells (Figure 1A). The differential proteomic analysis data showed that expression of $\mathrm{NADH}$ dehydrogenase (ubiquinone) iron-sulfur protein 5 (NDUFS5), subunit of respiratory chain complex I, reduced for 1.5 times, while the expression of succinate dehydrogenase (Complex II) has no significant difference between FH knockdown HK-2 cells and NC cells. Rieske iron-sulfur protein (RISP) and Cyt b-c1 complex subunit 6 (Complex III) reduced 1.5 fold in FH knockdown HK-2 cells. Meanwhile, the expression of OGDH and B5R (cytochrome b5 reductase) increased 1.5 and 1.7 fold. Except these defined ROS generation sites, $F_{1} F_{0}$ ATP synthase subunits ATP5A1, ATP5C1, ATP5I and ATP5L reduced by 1.7, 1.4, 1.5 and 2.1 times and ATP level reduced significantly (Figure 1C). Briefly, NOX, $\alpha-O G D H, B 5 R$, ATPase and Complex I and III would be the main sites of ROS formation in FH knockdown HK-2 cells.

\section{Glutathione metabolism and antioxidant systems in FH knockdown HK-2 cells}

The redox state of the cell is finely regulated by antioxidant systems, which include antioxidant enzymes and small molecular weight antioxidants. The proteomic analysis had identified the expression of all key enzymes involved in glutathione metabolism, increased their protein expression, which included Gpx, GR, GST, GS, G6PD and 6PGD (Table EV5), meanwhile, the activities of G6PD, 6PGD, GR, GST, GPx also increased compared with NC cells (Figure1 B, D, E, F). By contrast, there was no significant difference in the expression of glutamate cysteine ligase (GCL). In addition, the expression of other antioxidant enzymes, SOD2, NNTH (nicotinamide nucleotide transhydrogenase) and IDH2 increased 1.69, 1.77, 1.43 fold, however, the CAT, SOD1 
had no significant difference between FH knockdown HK-2 cells and NC cells. The expression of SOD2, IDH1 and IDH2 was further confirmed by western blotting

(Figure EV2). Furthermore, the expression of L-lactate dehydrogenase (LDHB), NADPH-dependent alcohol dehydrogenase (AKR1A1), glutathione dependent formaldehyde dehydrogenase (ADH5) and L-glutamate gamma semialdehyde dehydrogenase (ALDH7A1) increased 1.7, 1.5, 1.5, 1.4 fold compared with NC cells. These data suggested the increased activity of dehydrogenase would supply more antioxidants such as GSH, NADH or NADPH. Unfortunately, the content of GSH reduced dramatically, especially the ratio of GSH/GSSG decreased even if the content of NADPH increased compared with NC cells. The GSH synthesis substrate, glutamate, glycine and L-cysteine reduced significantly too (Figure $2 \mathrm{~A}-\mathrm{F}$ ).

\section{Key enzymes involved in renal redox metabolism of Dahl SS rats}

The FH activity was insufficient in renal cortex and medulla of SS rats compared with $\mathrm{SS} .13^{\mathrm{BN}}$. To confirm that the renal cells exhibit the same response to the insufficient $\mathrm{FH}$ activity as $\mathrm{FH}$ knockdown HK-2 cells, the expression and activity of these key enzymes involved in redox metabolism were detected. For ROS formation, the activity of NOX had been reported increase definitely in renal medulla of SS rats [5], similar to FH knockdown HK-2 cells. The expression of OGDH increased significantly, and the expression of ATP5A1 reduced significantly in renal medulla

(Figure 3). SS rats mitochondrial respiratory chain complex I and III had been reported involved in ROS production owing to the insufficient expression of EFTu (elongation factor Tu) protein in the mTAL mitochondria [12]. 

and medulla (Figure 4B). The ratio of GSH/GSSG reduced significantly in renal medulla $(P<0.01)$, and renal cortex $(P=0.065)$ (Figure $4 C)$. The GSH synthesis substrate glutamate, glycine and L-cysteine reduced significantly in renal medulla (Figure 4D).

Meanwhile, the activity of G6PD increased in renal cortex and medulla, but the redox metabolism to Fh knockdown HK2 cells.

\section{Redox state in kidney with SS rat oral administration of fumarate and malate}


132

and MDA increased dramatically in renal medulla and the oral administration of malate could attenuate significantly the increasing of $\mathrm{H}_{2} \mathrm{O}_{2}, \mathrm{MDA}$ and blood pressure caused by high salt diet (Figure $5 \mathrm{D} \mathrm{E}$ ). These data demonstrated that the substrate of $\mathrm{FH}$, fumarate, could exacerbate ROS production and hypertension in SS rats, on the contrary, the product of $\mathrm{FH}$, L-malate, could attenuate the hypertension induced by high salt diet.

\section{Discussion}

\section{Imbalance of redox state in FH knockdown HK-2 cells}

GST catalyzes the conjugation of the reduced form of GSH to xenobiotic substrates for the purpose of detoxification. The upregulated expression and improved activity of GPx and GST suggested excessive consumption of GSH after FH knockdown.

GR catalyzes the reduction of GSSG to the sulfhydryl form GSH via the conversion of $\mathrm{NADPH}$ to $\mathrm{NADP}^{+}$, which is a critical molecule in resisting oxidative stress and maintaining the reducing environment of the cell. The main source of NADPH in the cell is from the pentose phosphate pathway (PPP) in which G6PD and 6PGD were defined as the rate-limiting enzyme. The activity of GR, G6PD and 6PGD was increased significantly as a response to FH knockdown in HK-2 cells (Figure 1). It was also consistent with the increased protein expression. Except PPP, NADPH also can be produced from other oxidoreductases, such as IDH2, which oxidizes a substrate by reducing an electron acceptor usually as $\mathrm{NAD}^{+}$or $\mathrm{NADP}^{+}$and producing $\mathrm{NADH}$ or 
153

$\mathrm{NADPH}$. Indeed, the activity of $\mathrm{NADP}^{+}-\mathrm{IDH}_{2}$ increased significantly (Figure $1 \mathrm{C}$ ).

Meanwhile, the expression of other dehydrogenase such as LDHB, AKR1A1, ADH5, ALDH7A1 also increased significantly in Fh knockdown cells. These data suggested increasing the expression of dehydrogenase could provide more NADPH or NADH for the consumption of GR. In addition, oxidation of NADPH through mitochondrial electron transfer chains was decreased, owing to adrenodoxin oxidoreductase (FDXR), serving as the first electron transfer protein in all the mitochondrial P450 systems, reduced significantly. The ratio of $\mathrm{NADPH} \mathrm{NADP}^{+}$slightly increased (Figure $2 \mathrm{~B}$ ).

As all key enzymes (GR, GS, GST, GPx, G6PD and 6PGD) involved in glutathione metabolism increased their activities or protein expression, suggested that the oxidation-reduction reaction that GSH participated in was extremely active, excessive ROS and reducing molecular produced in HK-2 cells at the same time after $\mathrm{FH}$ knockdown.

The ratio of GSH/GSSG is often used as an indicator of the cellular redox state since it is the most abundant thiol-disulfide redox buffer in the cell [13]. Normally, GSH is about $30-100$ fold excess over GSSG. The oxidation of only a small amount of GSH to GSSG can significantly change the ratio and the redox status of the cell. However, knockdown of $\mathrm{FH}$ expression resulted in a significant decreasing of GSH/GSSG in HK-2 cells (Figure 2E). The results further confirmed that the insufficient FH activity could result in the oxidative stress. Nevertheless, where did these ROS come from?

The activity of NADPH oxidases was detected and showed significantly increased 
175

in FH knockdown HK-2 cells, which should be the contributor of ROS production

(Figure 1A). Mitochondrial enzymes are so far known to generate ROS including the TCA cycle enzymes aconitase (ACO), OGDH, PDH, GPDH (glycerol-3-phosphate dehydrogenase), B5R and Complexes I, II and III [14]. Current differential proteomics analysis data showed that the expression of NDUFS5, an important member of the Complex I, reduced significantly. Its content of expression modulates the efficiency of mitochondrial electron transport chain and ATP level [15]. The fully assembling of Complex I will be disturbed by insufficient expression of NDUFS5, the ROS generation will be promoted $[16,17]$. RISP as one of the catalytic subunits of Complex III, it had been demonstrated that deficiency of RISP influenced the stability of Complex I, Complex III and increased generation of ROS [18]. Both RISP and Cyt b-c1 complex subunit 6 reduced 1.5 fold in FH knockdown HK-2 cells. It suggested complex III contributed to the production of ROS. Meanwhile, the upregulation of both OGDH and B5R also promoted ROS production. ATP synthase had been defined as one important site of ROS generation. Its activity can modulate ROS formation and ATP level in mitochondria [19]. ATP5A1 was identified as a target for calpain-1 in diabetic hearts, leading to its proteolytic degradation, disruption of ATP5A1 may compromise mitochondrial function, resulting in excessive ROS generation in mice [20]. Current data showed that the expression of ATP synthase subunit ATP5A1, ATP5C1, ATP5I and ATP5 $L$ reduced significantly, and accordingly ATP level reduced too (Figure 1B). Briefly, the signature of redox metabolism for FH knockdown HK-2 cells would be NOX, $\alpha-O G D H, B 5 R$, ATPase and complex I, III worked as the main sites of ROS 
197

formation, and upregulation expression of GR, GS, GST, GPx, G6PD, 6PGD, SOD2, IDH2 and NNTH worked as an antagonistic response to the excessive ROS. The final substantial drop in GSH/GSSG ratio was the result of FH knockdown. The redox metabolism for FH knockdown HK-2 cells was summarized in Figure EV5.

\section{The oxidative stress in renal medulla and cortex of SS rats}

It had been demonstrated that NOX was the major source of excess superoxide produced in renal medulla of SS rats [5]. The increase of NOX activity is consistent with it in FH knockout HK-2 cells. The expression of OGDH increased in renal medulla, on the contrary, the expression of ATP5A1 reduced significantly in renal medulla and cortex of SS rats compared with SS.13 ${ }^{\mathrm{BN}}$. These results suggested that OGDH and ATPase may also contribute to ROS formation in renal medulla of Dahl SS rats. Meanwhile, one mitochondrial proteomics analysis also suggested that Complex I and Complex III were also the ROS production sites in renal medullary thick ascending limb (mTAL) of the SS rats [12]. Briefly, B5R, NOX, $\alpha-O G D H$, ATPase, Complex I and Complex III would be the sites of ROS production in renal medulla of SS rats.

For the antioxidant response of SS rats, the synthesis of GSH was also modulated by the insufficient substrates, as the levels of glycine, glutamate and cysteine were reduced in renal medulla compared with SS.13 ${ }^{\mathrm{BN}}$. The levels of GSH, GSSG and total GSH (GSH+GSSG) in renal medulla of SS rats were the similar to those in $\mathrm{FH}$ knockdown HK-2 cells. Especially, the ratio of GSH/GSSG also reduced significantly. GPx had been reported increase in SS medulla [21]. Other dehydrogenases such as 
IDH2, 6GPD and G6PD also increased their expression or activity. These enzymes in renal medulla of SS rats had the same response to oxidant stress as FH knockout HK-2 cells. The full comparison involved in redox balance between SS rats and FH knockout HK-2 cells was listed in Table EV5. All the data suggested that the imbalance of redox metabolism in renal medulla of SS rats was highly similar to FH knockout HK-2 cells.

\section{Fumarate and malate contribute to the imbalance of redox and hypertension in SS}

rats

There is no evidence to confirm that $\mathrm{FH}$ is the site of ROS production, but the excessive fumarate formed by the insufficient $\mathrm{FH}$ activity could lead to oxidative stress by succination of GSH [22]. This covalent adduct between fumarate and GSH reduced the efficiency and level of GSH in the cell. Meanwhile, the total GSH (GSH+GSSG), GSH reduced significantly in SS renal cells and HK-2 cells as inadequate synthetic substrate, glycine and glutamate. Obviously, the insufficient GSH flux attenuated the antioxidant ability of GSH system and promote ROS formation. Indeed, the content of fumarate had been proved to increase in SS renal medulla, increased the levels of $\mathrm{H}_{2} \mathrm{O}_{2}$ and MDA, and exacerbated the elevation of MAP (Figure 5). As the production of $\mathrm{FH}$, excess malate oral administration could reduce the generation of ROS and attenuated the high salt diet induced hypertension. The mechanism of malate working is mainly through increasing synthesis of arginine and NO [11]. 


\section{Conclusion}

The insufficient FH activity led to the accumulation of fumarate in renal medulla

respiratory chain complex I, III. The increased G6PD, 6PGD, IDH and other

dehydrogenase activity became the main antagonistic response of cells to oxidative

stress induced by FH insufficiency. Glutathione metabolism, as the most important

antioxidant mechanism, lost its balance due to insufficient synthesis substrate and

247 resulted in the decreasing of antioxidant capacity, which made the cells under the

248 oxidative stress state. Therefore, the defect of FH gene located on chromosome 13 of

249 SS rats cause a wide range of changes at several sites of ROS production and

250 antioxidant mechanisms

\section{Materials and Methods}

\section{Cell culture and siRNA-mediated RNA interference}

253 HK-2, a human kidney epithelial cell line was obtained from and cultured as analyses were performed 64 hours after transfection.

\section{Western blotting}


261

262

263

264

265

266

dilutions of primary antibodies used were Fumarase, FH (CST, USA) 1:1000;

Superoxide dismutase [Mn], SOD2 (Wanleibio, China) 1:2000; NADP'-dependent isocitrate dehydrogenase cytoplasmic, NADP ${ }^{+}-$IDH1 (Wanleibio, China) 1:2000; $\mathrm{NADP}^{+}$-dependent isocitrate dehydrogenase, mitochondrial, NADP ${ }^{+}-\mathrm{IDH} 2$ (BOSTER, China) 1:2000; Heat shock protein HSP 90, HSP90 (Wanleibio, China) 1:2000; 2-Oxoglutarate dehydrogenase, OGDH (CST, USA) 1:1000; ATP synthase subunit alpha, ATP5A1 (Proteintech, USA) 1:2000; $\beta$-Actin (CW0096, China) 1:10000. Secondary antibodies were HRP-conjugated goat anti-mouse IgG (BOSTER, China) or HRP-conjugated goat anti-rabbit IgG antibody (Santa Cruz, USA). Signals were developed with Thermo Super Signal West Pico Trial Kit. The intensities of the target proteins were quantified using Image J software and $\beta$-actin was used as loading control.

\section{Measurement of ROS and $\mathrm{H}_{2} \mathrm{O}_{2}$}

ROS level was detected using dichlorodihydrofluorescein diacetate $\left(\mathrm{H}_{2} \mathrm{DCF}-\mathrm{DA}\right)$ (Sigma-Aldrich) [25]. Fluorescence was determined with a fluorescence spectrometer (TECAN Infinite M200 PRO, Schweiz) at $485 \mathrm{~nm}$ (excitation) and 538 $\mathrm{nm}$ (emission). Cellular oxidant levels were expressed as relative DCF fluorescence per sample protein amounts (measured using a $\mathrm{BCA}$ assay). $\mathrm{H}_{2} \mathrm{O}_{2}$ generation was detected using Hydrogen Peroxide assay kit (Jiancheng Biochemical).

\section{Proteomic analysis}

(1) Protein reduce, cysteine block and digest

HK-2 cells were lysed in a buffer containing $9 \mathrm{~mol} / \mathrm{L}$ Urea, 4\% CHAPS, $1 \%$ DTT, 
1\% IPG buffer (GE Healthcare) at $4{ }^{\circ} \mathrm{C}$ for $1 \mathrm{~h}$. The cell lysates were centrifuged at $15000 \mathrm{~g}$ for $25 \mathrm{~min}$. The supernatant were collected and the protein concentration was determined by Bradford method [26]. A volume corresponding to $100 \mu \mathrm{g}$ of protein were precipitated with 5 volumes of acetone at $-20^{\circ} \mathrm{C}$ for $1 \mathrm{~h}$. After centrifugation for $10 \mathrm{~min}$ at $15000 \mathrm{~g}$, the deposits were collected and dried by vacuum freezing dryer. Then dissolve protein pellets in $50 \mu \mathrm{L}$ iTRAQ dissolution buffer (Applied Biosystems) and add $4 \mu \mathrm{L}$ Reducing Reagent (Applied Biosystems) at $60{ }^{\circ} \mathrm{C}$ for $1 \mathrm{~h}$. After cooling samples to room temperature (RT), cysteine residues were blocked with $2 \mu \mathrm{L} 200 \mathrm{mmol} / \mathrm{L}$ methylmethanethiosulfate by incubating at RT for $10 \mathrm{~min}$. The protein solutions were added to $10 \mathrm{kDa}$ ultrafiltration tubes and cleaned by centrifugation (12000 rpm, $20 \mathrm{~min}$ ). Then $100 \mu \mathrm{L}$ iTRAQ dissolution buffer was added in ultrafiltration tubes, centrifuged at $12000 \mathrm{~g}$ for $15 \mathrm{~min}$ and repeat this step three times. Place the column in a new tube, add $50 \mu \mathrm{L}$ sequencing-grade trypsin $(50 \mathrm{ng} / \mu \mathrm{L})$ and incubate at $37{ }^{\circ} \mathrm{C}$ for 12 hours. Afterward, centrifuge by $12000 \mathrm{~g}$ for $20 \mathrm{~min}$ and collect the peptide. Transfer the filter units to new collection tube and add $50 \mu \mathrm{L}$ iTRAQ dissolution buffers to centrifuge the tube again. Combined the two filter solutions.

(2) Protein digestion and labeling with iTRAQ reagents

The labeling reactions were performed following the manufacturer's recommendations. Peptide samples from negative control HK-2 cells were labeled with iTRAQ reagent, 113 isobaric tag and peptide samples from FH siRNA-treated HK-2 cells were labeled by adding the same amount of iTRAQ reagent, 114 isobaric 
tag. After $2 \mathrm{~h}$ of incubation at RT, reactions were stopped by adding $100 \mu \mathrm{L}$ of water, to each vial.

(3) Peptide fractionation by strong cation exchange chromatography The mixed peptides were fractionated by strong cation exchange chromatography (SCX). Samples were separated using an Agilent 1200 HPLC System (Agilent), Michrom column (Poly-SEA $5 \mu 300 \AA 2.0 \times 150 \mathrm{~mm}$ ) at a flow rate of 0.3 $\mathrm{ml} / \mathrm{min}$, using a nonlinear binary gradient starting with buffer $\mathrm{A}$ (10 mmol/L formic acid, $20 \%$ acetonitrile) and transitioning to buffer $B(500 \mathrm{mmol} / \mathrm{L}$ formic acid, $20 \%$ acetonitrile). First, the column was washed with buffer $\mathrm{A}$ for $5 \mathrm{~min}$ and peptides were eluted with a four-step gradient: first a linear gradient of 5-50\% buffer B for 25 min, followed by a linear gradient of $50-80 \%$ buffer B for 5 min. The gradient was ramped to $100 \%$ buffer $B$ in $1 \mathrm{~min}$, and held for $10 \mathrm{~min}$. Collect the first segment from 0-5 min, then collect each segment with 4 min interval for the 6-44 min, and for the last segment from $45-50$ min, with a total of 12 segments. Dry every segment in a vacuum freezing dryer for LC-MSMS analysis.

(4) RPLC-MS/MS analysis

The online Nano-RPLC was employed on the Eksigent nanoLC-Ultra ${ }^{\mathrm{TM}}$ 2D System (AB SCIEX). The samples were loaded on $C_{18}$ nano LC trap column (100 $\mu \mathrm{m} \times 3$ $\mathrm{cm}, \mathrm{C}_{18}, 3 \mu \mathrm{m}, 150 \AA$ ) and washed by Nano-RPLC Buffer A (0.1\% formic acid, $2 \%$ acetonitrile) at $2 \mu \mathrm{L} / \mathrm{min}$ for $10 \mathrm{~min}$. An elution gradient of $5-35 \%$ acetonitrile $(0.1 \%$ formic acid) in 70 min gradient was used on an analytical Chrom XP $\mathrm{C}_{18}$ column (75 $\mu \mathrm{m} \times 15 \mathrm{~cm}, \mathrm{C}_{18}, 3 \mu \mathrm{m} 120 \AA$ ) with spray tip. Data acquisition was performed with a 
Triple TOF 5600 System (AB SCIEX, USA) fitted with a Nanospray III source (AB SCIEX, USA) and a pulled quartz tip as the emitter (New Objectives, USA). Data were acquired using an ion spray voltage of $2.5 \mathrm{kV}$, curtain gas of $30 \mathrm{PSI}$, nebulizer gas of $5 \mathrm{PSI}$, and an interface heater temperature of $150{ }^{\circ} \mathrm{C}$. For information-dependent acquistion (IDA), survey scans were acquired in $250 \mathrm{~ms}$ and as many as 35 product ion scans were collected if they exceeded a threshold of 150 counts per second (counts/s) with a $2^{+}$to $5^{+}$charge-state. The total cycle time was fixed to $2.5 \mathrm{~s}$. A rolling collision energy setting was applied to all precursor ions for collision-induced dissociation (CID). Dynamic exclusion was set for $1 / 2$ of peak width (18 s), and the precursor was then refreshed off the exclusion list.

(5) Protein identification and quantification

Data were processed with Protein Pilot Software v. 4.0 (AB SCIEX, USA) against Homo sapiens database using the Paragon algorithm [27]. Protein identification was performed with the search option: emphasis on biological modifications. The database search parameters were as following: instrument was Triple TOF 5600, iTRAQ 2-plex quantification, cysteine modified with iodoacetamide, biological modifications were selected as the ID focus, trypsin digestion. An automatic decoy database search strategy was employed to estimate the false discovery rate (FDR) using the PSPEP (Proteomics System Performance Evaluation Pipeline Software, integrated in the Protein Pilot Software). The FDR was calculated as the false positive matches divided by the total matches. The ITRAQ 2-plex was chosen for protein quantification with unique peptides during the search. A total of 3174 
proteins were identified in HK-2 cells, with the value of global FDR from fit less than $1 \%$ and identified with more than two peptides for $95 \%$ confidence were considered for further analysis. Proteins with a fold change of $>1.50$ or $<0.67$ were considered to be significantly differentially expressed.

\section{GO and KEGG pathway enrichment analysis}

$$
\text { The multi-omics data analysis tool, Omics Bean, was used to analyze the }
$$
obtained proteomics data (http://www.omicsbean.com:88), in which distributions in biological functions, subcellular locations and molecular functions were assigned to each protein based on Gene Ontology (GO) categories. The Kyoto Encyclopedia of Genes and Genomes (KEGG) pathway analysis was performed to enrich high-level functions in the defined biological systems.

\section{Enzymes activities assays}

The obtained pellets were lysed by three freeze-thaw cycles to ensure that the mitochondrial membrane was disrupted and enzymes were accessible. The protein content was determined with BCA Protein Assay Kit (Beyotime Institute of Biotechnology, China).

The enzyme activity of FH was measured based on a previous reported method [8]. The activities of glutathione reducatase (GR) were assayed using the Beyotime Detection kits (Beyotime Institute of Biotechnology, China) according to the corresponding protocols. The activities of glutathione S-transferase (GST), glutathione peroxidase (GPx) in HK-2 cells were assayed using the Jian cheng Biochemical detection kits according to the standard protocols. 
371 Glucose-6-phosphate dehydrogenase (G6PD) and 6-phosphogluconate

372 dehydrogenase (6PGD) activities assay were performed as described previously [28,

373 29]. Isocitrate dehydrogenase, cytoplasmic (NADP $\left.{ }^{+}-I_{D H 1}\right)$ and isocitrate

374 dehydrogenase, mitochondrial $\left(\mathrm{NADP}^{+}-\mathrm{IDH} 2\right)$ are $\mathrm{NADP}^{+}$-dependent isocitrate

375 dehydrogenase (NADP $\left.{ }^{+}-\mathrm{IDH}\right)$. NADP ${ }^{+}-\mathrm{IDH}$ activity was assayed by

376 spectrophotometric monitoring of the reduction of $\mathrm{NADP}^{+}$to $\mathrm{NADPH}$ at $340 \mathrm{~nm}$

377 according to protocol by Connie S. Yarian [30]. The NADPH oxidase activity was

378 determined using the NADPH oxidase assay kit purchased from Nanjing Jiangcheng

379 Bioengineering Institute according to the manufacturer's instructions.

380

381

382

\section{GSH (GSSG) and NADPH (NADP ${ }^{+}$) measurements}

The determination of reduced glutathione (GSH) and oxidized glutathione (GSSG) were performed using DTNB method with kits according to the corresponding kit protocols (Beyotime, China). The determination of $\mathrm{NADP}^{+}$and NADPH were performed with kits according to the corresponding kit protocols (Comin, China).

\section{GC-MS assay}

The extraction of metabolites in tissue and cells samples was performed according to the method described in previous reports [31, 32]. Dried tissue and cell residues were derivatized using a two-step procedure with a minor modification [33, 34]. For oximation, $80 \mu \mathrm{L}$ of $10 \mathrm{mg} / \mathrm{mL}$ methoxyamine hydrochloride (Sigma-Aldrich) dissolved in pyridine was mixed with a lyophilized sample, and kept at $30^{\circ} \mathrm{C}$ for 90 min. Then, $80 \mu \mathrm{L}$ of N,O-bis(trimethylsilyl)-trifluoroacetamide (BSTFA) with 1\% TMCS 
(Sigma-Aldrich) was added for derivatization, and heated to $70^{\circ} \mathrm{C}$ for 60 minutes.

GC-MS analysis was performed according to our previous report [33], using a 7890A GC/5975C Inert MSD (Agilent Technologies, Wilmington, DE), coupled with a DB-5 column (30 m × $0.25 \mathrm{~mm}$ I.D.; film thickness: $0.25 \mu \mathrm{m}$; Agilent J\&W Scientific, USA). Helium was used as carrier gas at a constant flow rate of $1 \mathrm{ml} / \mathrm{min}$. The GC temperature programming was set to 2 min isothermal heating at $80^{\circ} \mathrm{C}$, followed by $10^{\circ} \mathrm{C} / \mathrm{min}$ oven temperature ramps to $120^{\circ} \mathrm{C}, 5^{\circ} \mathrm{C} / \mathrm{min}$ to $260^{\circ} \mathrm{C}$, and then increased at a rate of $10{ }^{\circ} \mathrm{C} / \mathrm{min}$ to $300^{\circ} \mathrm{C}$, where it was held for $2 \mathrm{~min}$. Fumarate, L-malate, glutamate, glycine, L-cysteine standards were analyzed under identical experimental conditions for further identification. One $\mu \mathrm{L}$ of the final derivatized aliquots was injected into the GC-MS.

\section{Measurement of ATP}

The ATP assay was performed with a kit from Promega (G7570) according to manufacturer's instruction. The luminescence was recorded in a TECAN Infinite M200 PRO (Schweiz) with an integration time of 5 s per well.

\section{Animals and tissues}

Male SS rats (SS/JrHsdMcwi) and consomic, salt-insensitive SS.13 ${ }^{\mathrm{BN}}$ rats [35] were bred in a pathogen-free animal house and maintained on a purified AIN-76A rodent diet (Dyets) containing $0.4 \% \mathrm{NaCl}$ with free access to water. For tissue collection, kidneys were flushed in situ with cold saline and the renal cortex and medulla were quickly removed and snap-frozen in liquid nitrogen and stored at $-80^{\circ} \mathrm{C}$. 
415

416

417

assays, Western blots and metabolite analysis. The experiments were approved by the Institutional Animal Ethics Committee of Xi'an Jiaotong University.

\section{Fumarate and malate supplementation}

Male SS rats were fed a $0.4 \% \mathrm{NaCl}$ diet since weaning. After three days of stable baseline blood pressure at approximately 7 weeks of age, rats were switched to an $8 \%$ $\mathrm{NaCl}$ diet (HS). Fumarate $(50 \mathrm{mg} / \mathrm{kg} / \mathrm{d})$ [36], malate $(600 \mathrm{mg} / \mathrm{kg} / \mathrm{d})$ [37], or an equal volume of distilled water was delivered by gavage. Serum and kidneys were harvested at the end of blood pressure measurement on day 17 of the HS diet.

\section{Measurement of blood pressure}

Blood pressure of conscious rats was measured using tail-cuff plethysmography with a CODA-4 computerized system (Kent Scientific Corporation, Torrington, Connecticut, USA). Mean arterial pressure (MAP) was measured between 14:00 and 16:00 by a single experienced operator. Rats were trained for the procedure for five consecutive days. After calibrating the tail-cuff apparatus, rats were placed on the platform and allowed to rest in a glass restrainer. A black conical plastic piece with a nose opening was placed over the head region of the rat to cover the eyes and allow the rat to rest. The platform was heated to $30-32{ }^{\circ} \mathrm{C}$ to increase the detection of the oscillation waveforms generated by the blood flow in the tail. Each session was composed of 15 successive measurements of the systolic and diastolic pressure, which were averaged. Heart rate was recorded at the same time.

\section{Measurement of $\mathrm{H}_{2} \mathrm{O}_{2}$ and MDA in the kidney of SS rats}

$\mathrm{H}_{2} \mathrm{O}_{2}$ and malondialdehyde (MDA) generation were detected using Hydrogen 
437

438

439

440

441

442

443

444

445

Peroxide assay kit (Jiancheng Biochemical).

\section{Statistical analysis}

All values are presented as mean \pm SEM. All data were analyzed using the Student's t-test or ANOVA analysis. Probabilities of $<0.05$ were considered to be statistically significant. All of the statistical tests were performed with the Graph Pad Prism software, version 6.0 (Graph Pad Software Inc., San Diego, CA).

Acknowledgments: This study was supported in part by the National Natural Science Foundation of China (Grant No. 81570655, 81770728, 51703178)

\section{Conflict of interest}

The authors declare that they have no conflict of interest.

\section{References}

1. Kotchen TA, Jr CA, Liang M (2016) Ushering Hypertension Into a New Era of Precision Medicine. Jama 315: 1-2

2. Whelton PK (2015) The Elusiveness of Population-Wide High Blood Pressure Control. Annual Review of Public Health 36: 109

3. Kearney PM, Whelton M, Reynolds K, Muntner P, Whelton PK, He J (2005) Global burden of hypertension: analysis of worldwide data. Lancet 365: 217-223

4. Cowley AW, Jr., Roman RJ, Kaldunski ML, Dumas P, Dickhout JG, Greene AS, Jacob HJ (2001) Brown Norway chromosome 13 confers protection from high salt to consomic Dahl S rat. Hypertension 37: 456-61

5. Taylor NE, Glocka P, Liang M, Cowley AW, Jr. (2006) NADPH oxidase in the renal medulla causes oxidative stress and contributes to salt-sensitive hypertension in 
459

460

461

462

463

464

465

466

467

468

469

470

471

472

473

474

475

476

477

478

479

480

Dahl S rats. Hypertension 47: 692-8

6. Taylor NE, Cowley AW, Jr. (2005) Effect of renal medullary H2O2 on salt-induced hypertension and renal injury. American journal of physiology Regulatory, integrative and comparative physiology 289: R1573-9

7. Jr CA (2008) Renal medullary oxidative stress, pressure-natriuresis, and hypertension. Hypertension 52: 777-86

8. Tian Z, Liu Y, Usa K, Mladinov D, Fang Y, Ding X, Greene AS, Cowley AW, Jr., Liang M (2009) Novel role of fumarate metabolism in dahl-salt sensitive hypertension. Hypertension 54: 255-60

9. Tian Z, Greene AS, Usa K, Matus IR, Bauwens J, Pietrusz JL, Cowley AW, Jr., Liang M (2008) Renal regional proteomes in young Dahl salt-sensitive rats. Hypertension

51: 899-904

10. Usa K, Liu Y, Geurts AM, Cheng Y, Lazar J, Baker MA, Grzybowski M, He Y, Tian Z, Liang M (2017) Elevation of fumarase attenuates hypertension and can result from a nonsynonymous sequence variation or increased expression depending on rat strain. Physiological genomics 49: 496-504

11. Hou E, Sun N, Zhang F, Zhao C, Usa K, Liang M, Tian Z (2017) Malate and Aspartate Increase L-Arginine and Nitric Oxide and Attenuate Hypertension. Cell reports 19: 1631-1639

12. Zheleznova NN, Yang C, Ryan RP, Halligan BD, Liang M, Greene AS, Cowley AW, Jr. (2012) Mitochondrial proteomic analysis reveals deficiencies in oxygen utilization in medullary thick ascending limb of Henle in the Dahl salt-sensitive rat. Physiological 
481

482

483

484

485

486

487

488

489

490

491

492

493

494

495

496

497

498

499

500

501

502

genomics 44: 829-42

13. Schafer FQ, Buettner GR (2001) Redox environment of the cell as viewed through the redox state of the glutathione disulfide/glutathione couple. Free radical biology \& medicine 30: 1191-212

14. Lin MT, Beal MF (2006) Mitochondrial dysfunction and oxidative stress in neurodegenerative diseases. Nature 443, 787-795. Nature 443: 787-795

15. Siengdee P, Trakooljul N, Murani E, Schwerin M, Wimmers K, Ponsuksili S (2015)

MicroRNAs Regulate Cellular ATP Levels by Targeting Mitochondrial Energy

Metabolism Genes during C2C12 Myoblast Differentiation. PloS one 10: e0127850

16. Schulte U, Fecke W, Krüll C, Nehls U, Schmiede A, Schneider R, Ohnishi T, Weiss

$\mathrm{H}$ (1994) In vivo dissection of the mitochondrial respiratory NADH: ubiquinone oxidoreductase (complex I). Biochimica et biophysica acta 1187: 121-4

17. Dunning CR, McKenzie M, Sugiana C, Lazarou M, Silke J, Connelly A, Fletcher JM, Kirby DM, Thorburn DR, Ryan MT (2007) Human CIA30 is involved in the early assembly of mitochondrial complex I and mutations in its gene cause disease. The EMBO journal 26: 3227-37

18. Diaz F, Enríquez JA, Moraes CT (2012) Cells Lacking Rieske Iron-Sulfur Protein Have a Reactive Oxygen Species-Associated Decrease in Respiratory Complexes I and IV. Molecular \& Cellular Biology 32: 415-29

19. Nina K, Valentina G (2016) The Dual Function of Reactive Oxygen/Nitrogen Species in Bioenergetics and Cell Death: The Role of ATP Synthase. Oxidative Medicine \& Cellular Longevity 2016: 3869610 
503

504

505

506

507

508

509

510

511

512

513

514

515

516

517

518

519

520

521

522

523

524

20. Ni R, Zheng D, Xiong S, Hill DJ, Sun T, Gardiner RB, Fan GC, Lu Y, Abel ED, Greer PA (2016) Mitochondrial calpain-1 disrupts ATP synthase and induces superoxide generation in type-1 diabetic hearts: a novel mechanism contributing to diabetic cardiomyopathy. Diabetes 65: 255-68

21. Taylor NE, Maier KG, Roman RJ, Jr CA (2006) NO synthase uncoupling in the kidney of Dahl S rats: role of dihydrobiopterin. Hypertension 48: 1066-1071

22. Zheng L, Cardaci S, Jerby L, Mackenzie ED, Sciacovelli M, Johnson TI, Gaude E, King A, Leach JD, Edrada-Ebel R (2015) Fumarate induces redox-dependent senescence by modifying glutathione metabolism. Nature Communications 6: 6001-6001

23. Liang M, Pietrusz JL (2007) Thiol-related genes in diabetic complications: a novel protective role for endogenous thioredoxin 2. Arteriosclerosis Thrombosis \& Vascular Biology 27: 77-83

24. Di F, Chun Y, Geurts AM, Terry K, Mingyu L, Jozef L, Mattson DL, O'Connor PM, Cowley AW (2012) Increased expression of NAD(P)H oxidase subunit p67(phox) in the renal medulla contributes to excess oxidative stress and salt-sensitive hypertension. Cell Metabolism 15: 201-208

25. LeBel CP, Ischiropoulos H, Bondy SC (1992) Evaluation of the probe $2^{\prime}, 7^{\prime}$-dichlorofluorescin as an indicator of reactive oxygen species formation and oxidative stress. Chemical research in toxicology 5: 227-31

26. Bradford MM (1976) RAPID AND SENSITIVE METHOD FOR QUANTITATION OF MICROGRAM QUANTITIES OF PROTEIN UTILIZING PRINCIPLE OF PROTEIN-DYE 
BINDING. Analytical Biochemistry 72: 248-254

27. Shilov IV, Seymour SL, Patel AA, Loboda A, Tang WH, Keating SP, Hunter CL, Nuwaysir LM, Schaeffer DA (2007) The paragon algorithm, a next generation search engine that uses sequence temperature values and feature probabilities to identify peptides from tandem mass spectra. Molecular \& Cellular Proteomics 6: 1638-1655

28. Tian WN, Braunstein LD, Pang J, Stuhlmeier KM, Xi QC, Tian X, Stanton RC (1998) Importance of glucose-6-phosphate dehydrogenase activity for cell growth. The Journal of biological chemistry 273: 10609-17

29. Tian WN, Pignatare JN, Stanton RC (1994) Signal transduction proteins that associate with the platelet-derived growth factor (PDGF) receptor mediate the PDGF-induced release of glucose-6-phosphate dehydrogenase from permeabilized cells. The Journal of biological chemistry 269: 14798-805

30. Yarian CS, Toroser D, Sohal RS (2006) Aconitase is the main functional target of aging in the citric acid cycle of kidney mitochondria from mice. Mechanisms of Ageing \& Development 127: 79-84

31. Wu H, Xue R, Tang Z, Deng C, Liu T, Zeng H, Sun Y, Shen X (2010) Metabolomic investigation of gastric cancer tissue using gas chromatography/mass spectrometry. Analytical and Bioanalytical Chemistry 396: 1385-95

32. Chen F, Zhang J, Song X, Yang J, Li H, Tang H, Liao YC (2011) Combined metabonomic and quantitative real-time PCR analyses reveal systems metabolic changes of Fusarium graminearum induced by Tri5 gene deletion. Journal of Proteome Research 10: 2273-85 
547 33. Wang L, Hou E, Wang Z, Sun N, He L, Chen L, Liang M, Tian Z (2014) Analysis of metabolites in plasma reveals distinct metabolic features between Dahl

549 salt-sensitive rats and consomic SS. 13 BN rats. Biochemical and biophysical research communications 450: 863-869

552 (2005) Chemical derivatization and mass spectral libraries in metabolic profiling by Jacob HJ (2001) Brown Norway chromosome 13 confers protection from high salt to consomic Dahl S rat. Hypertension 37: 456-461 antagonist, on blood pressure and heart rate in conscious spontaneously hypertensive rats. Japanese Journal of Pharmacology 63: 121 
565

566

567

568

569

570

571

572

573

574

575

576

577

578

579

580

581

582

583

584

585

586

\section{Figure legends}

Figure 1 - NADPH oxidase and key enzymes involved in glutathione metabolism activities in fumarase insufficient HK-2 cells.

A NADPH oxidase activity enhanced in fumarase insufficiency HK-2 cells.

B Fumarase insufficiency attenuated ATP production.

C Glucose-6-phosphate 1-dehydrogenase (G6PD), 6-Phosphogluconate dehydrogenase (6PGD) and NADP ${ }^{+}-$IDH activity.

D Glutathione reductase (GR) activity.

E Glutathione S-transferase (GST) activity.

F Glutathione peroxidase (GPx) activity. Mock siRNA targeting fumarase that had no sequence homology with any mammalian genes as a negative control.

Data are presented as mean values \pm SEM, $n=4,{ }^{*} p<0.05,{ }^{*} p<0.01$ compared to negative control.

Figure 2 - Fumarase insufficiency is associated with increased NADPH, NADP ${ }^{+}$and reduced total GSH, GSH/GSSG ratio.

A NADPH and $\mathrm{NADP}^{+}$levels were detected in negative control and fumarase insufficient HK-2 cells.

B NADPH/NADP ${ }^{+}$ratio.

C Total GSH (GSH+GSSH) and GSH levels.

D GSSG levels.

E GSH/GSSG ratio. 
587 F Fold changes of the glutamate, glycine and L-cysteine levels in fumarase insufficient

HK-2 cells was calculated as a ratio relative to negative control. Mock siRNA targeting

589 fumarase that had no sequence homology with any mammalian genes as a negative

control. Data are presented as mean values $\pm \mathrm{SEM}, \mathrm{n}=6, * \mathrm{p}<0.05, * * \mathrm{p}<0.01$

591

compared to negative control.

592

Figure 3 - Western blot analysis of protein abundance of OGDH and ATP5A1 in the

594

595

596

597

598

599

600

601

602

603

604

605

606

607

608

kidney of SS and SS.13 ${ }^{\mathrm{BN}}$ rats.

A Abundance of $\alpha$-oxoglutarate dehydrogenase (OGDH) and ATP synthase subunit alpha (ATP5A1) in the renal cortex.

B Abundance of OGDH and ATP5A1 in the renal medulla. $\beta$-actin is used as the internal loading control. Data are presented as mean values $\pm S E M, n=4,{ }^{*} p<0.05$ compared to negative control.

Figure 4 - Total GSH levels and GSH/GSSG ratio were reduced in the kidney of SS rats.

A Total GSH (GSH+GSSH) and GSH levels reduced in the renal cortex and medulla.

B GSSG levels.

C GSH/GSSG ratio.

D Fold changes of the glutamate, glycine and L-cysteine in the renal medulla.

E G6PD, 6PGD activitives in the renal cortex and medulla.

F NADP ${ }^{+}-$IDH activity in the renal cortex and medulla.

Data are presented as mean values $\pm \mathrm{SEM}, \mathrm{n}=5, * \mathrm{p}<0.05$ compared to negative 
609

610

611

612

613

614

615

616

617

618

619

620

621

622

623

624

control.

Figure 5 - Levels of $\mathrm{H}_{2} \mathrm{O}_{2}$ and MAD in the kidney of SS rats with fumarate or malate supplementation.

A Mean arterial blood pressure (MAP) of SS rats on $0.4 \%$ salt (normal salt, NS) diet with or without malate (fumarate) or $8 \%$ salt (high salt, HS) diet with or without malate supplementation by gavage.

B Levels of $\mathrm{H}_{2} \mathrm{O}_{2}$ in the renal cortex and medulla of SS rats on $0.4 \%$ salt with or without malate (fumarate) supplementation by gavage.

C Levels of MDA in the renal cortex and medulla of SS rats on $0.4 \%$ salt with or without malate (fumarate) supplementation by gavage.

D Levels of $\mathrm{H}_{2} \mathrm{O}_{2}$ in the renal cortex and medulla of $\mathrm{SS}$ rats on $0.4 \%$ salt or $8 \%$ salt diet with or without malate supplementation by gavage.

E Levels of MDA in the renal cortex and medulla of SS rats on $0.4 \%$ salt or $8 \%$ salt diet with or without malate supplementation by gavage. Data are presented as mean values \pm SEM. $n=5-6,{ }^{*} p<0.05, * * p<0.01$ 
625

626

627

628

629

630

631

632

633

634

635

636

637

638

639

640

641

642

643

644

645

646

647

\section{Expanded View Figure legends}

Figure EV1 - Down-regulation of fumarase expression and activity is associated with the accumulation of fumarate, ROS and $\mathrm{H}_{2} \mathrm{O}_{2}$ in the $\mathrm{HK}-2$ cells.

A Western bloting densitometric analysis of fumarase of HK-2 cells on both negative control (NC) and FH-siRNA treated (FH-SiRNA) with the associated gel shown.

B Fumarase (FH) activity.

C Fold changes of fumarate and L-malate level in fumarase insufficient HK-2 cells were calculated as a ratio relative to negative control.

D ROS levels.

E, $\mathrm{H}_{2} \mathrm{O}_{2}$ levels. Mock siRNA targeting fumarase that had no sequence homology with any mammalian genes as a negative control.

Data are presented as mean values \pm SEM, $\mathrm{n}=4-6,{ }^{*} \mathrm{p}<0.05,{ }^{* *} \mathrm{p}<0.01$ compared to negative control.

Figure EV2 - Expressional changes of SOD2, IDH1, IDH2 and HSP90 in the HK-2 cells induced by fumarase insufficiency.

Left: a representative result of western blotting shows the expressions of SOD2, IDH1, IDH2, and HSP90 in fumarase insufficient HK-2 cells compared to negative control.

Right: histogram shows the expression levels of the four proteins in these HK-2 cells as determined by densitometric analysis.

$\beta$-actin is used as the internal loading control. Mock siRNA targeting fumarase that had no sequence homology with any mammalian genes as a negative control.

Data are presented as mean values \pm SEM. $n=4,{ }^{*} p<0.05,{ }^{* *} p<0.01$ significant difference 
648

649

650

651

652

653

654

655

656

657

658

659

660

661

662

663

664

665

666

667

668

669

670

671

672

673

674

675

from negative control.

Figure EV3 - GO annotation of identified significant changes proteins in three categories: biological process (BP), cellular component (CC) and molecular function (MF).

All of biological processes were ranked in term of the enrichment of the differentially expressed proteins, and the top 10 are presented.

Figure EV4 - Distribution of enriched KEGG Pathway. Top 10 enriched pathway are shown here.

$\mathrm{P}$ value $=0.01$ (red) and $\mathrm{P}$ value $=0.05$ (blue) as two selected cutoff are highlighted on the figure, as an indicator to show how significant the results are based on KEGG enrichment.

Figure EV5. Role of fumarase insufficiency in reactive oxygen species metabolism.

The proteins or compounds involved in ROS generation (red stars) and antioxidant defence (blue stars). The red arrows indicate proteins or compounds identified as significantly increased in our study. The green arrows indicate proteins or compounds identified as significantly decreased in our study. FH, Fumarase; OGDH, 2-oxoglutarate dehydrogenase; C I, C II and C III, the electron-transport chain (ETC) complexes I, II and III; B5R, cytochrome b5 reductase; NNTH, Nicotinamide nucleotide transhydrogenase; G6PD, Glucose-6-phosphate 1-dehydrogenase; 6PGD, 6-phosphogluconate dehydrogenase; IDH2, Isocitrate dehydrogenase [NADP+], mitochondrial; PRDX5, Peroxiredoxin-5, mitochondrial; FDXR, NADPH:adrenodoxin oxidoreductase, mitochondrial; SOD2, Superoxide dismutase [Mn]; GS, Glutathione synthetase; GR, Glutathione reductase; GPx, Glutathione peroxidase; GSH, reduced glutathione; GSSG, oxidized glutathione.PM, Plasma membrane; OMM, Outer mitochondrial membrane; IMM, Inner mitochondrial membrane. 


\section{Expanded View Table legends}

677 Table EV1. Summary of the total proteins were identified and quantified reliably by

678 iTRAQ.

679

680 Table EV2. Differentially expressed proteins in FH-siRNA treated HK-2 cell line

681 identified from iTRAQ analysis.

682

683 Table EV3. GO enrichment of the total significant changes proteins.

684

685 Table EV4. KEGG enrichment of the total significant changes proteins.

686

687 Table EV5. The enzymes and metabolites involved in ROS generation and

688 antioxidant defence in fumarase insufficient HK-2 cell and SS rats. 
bioRxiv preprint doi: https://doi.org/10.1101/302182; this version posted April 16, 2018. The copyright holder for this preprint (which was not certified by peer review) is the author/funder. This article is a US Government work. It is not subject to copyright under 17 USC 105 and is also made available for use under a CCO license.

\section{Insufficient fumarase contributes to generating reactive oxygen species in}

\section{Dahl salt sensitive rats}

4 Entai Hou ${ }^{\#}$, Xuewei Zheng1 ${ }^{\#}$, Zhe Yang ${ }^{1}$, Xian Li ${ }^{1}$, Zerong Liu ${ }^{1}$, Meng Chen ${ }^{1}$,

$6 \quad$ 1The Key Laboratory of Biomedical Information Engineering of Ministry of

7 Education, School of Life Science and Technology, Xi'an Jiaotong University,

$$
\text { Xi'an 710049, China }
$$

$9 \quad{ }^{2}$ Center of Systems Molecular Medicine, Department of Physiology, Medical 
bioRxiv preprint doi: https://doi.org/10.1101/302182; this version posted April 16, 2018. The copyright holder for this preprint (which was not certified by peer review) is the author/funder. This article is a US Government work. It is not subject to copyright under 17 USC 105 and is also

23 Figure 1

made available for use under a CCO license.

24

A

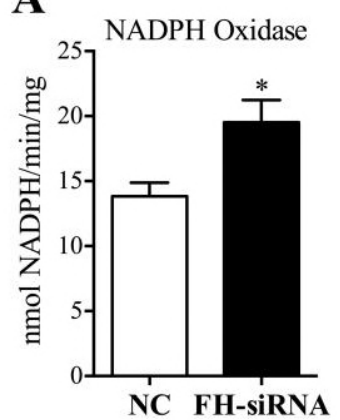

C

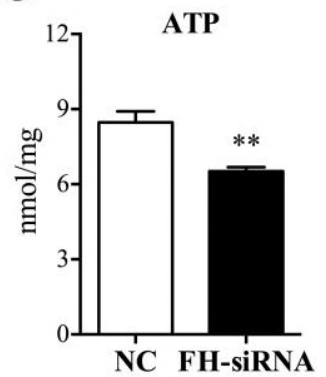

B

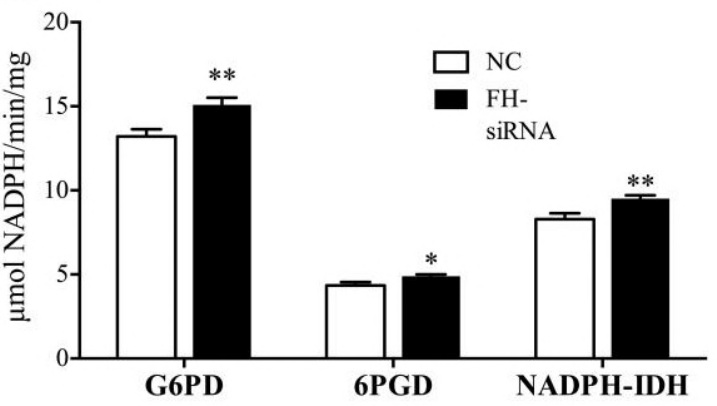

D
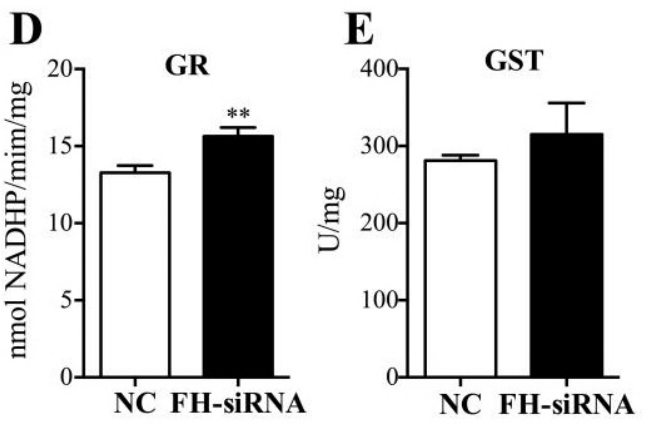

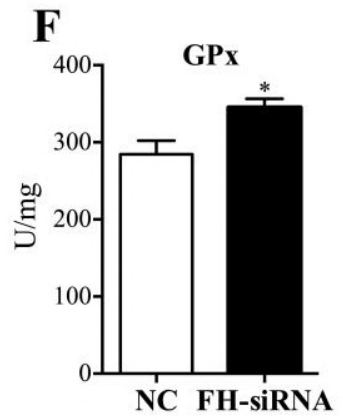

Figure 2

32
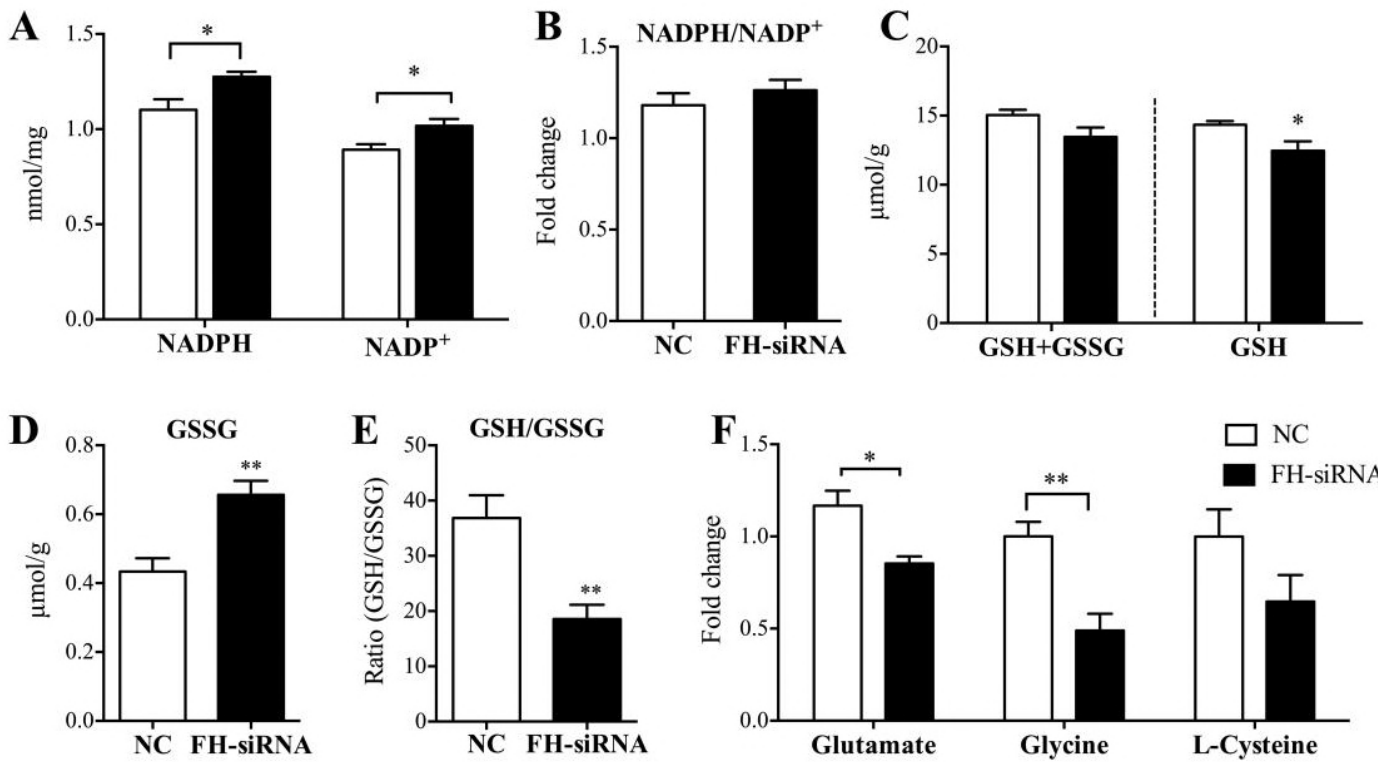
bioRxiv preprint doi: https://doi.org/10.1101/302182; this version posted April 16, 2018. The copyright holder for this preprint (which was not certified by peer review) is the author/funder. This article is a US Government work. It is not subject to copyright under 17 USC 105 and is also 36 made available for use under a CCO license.

$37 \quad$ Figure 3
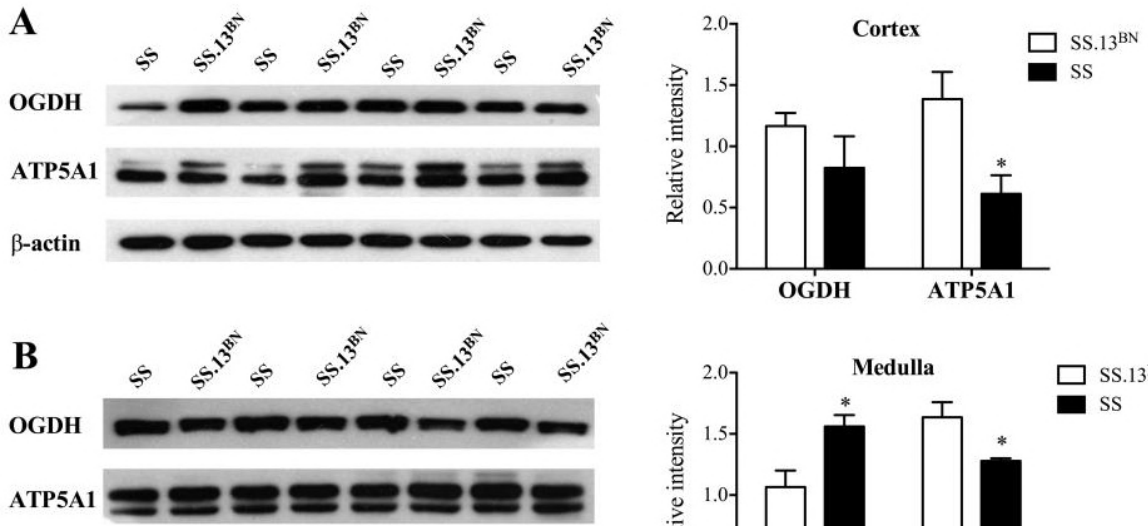

$\beta$-actin

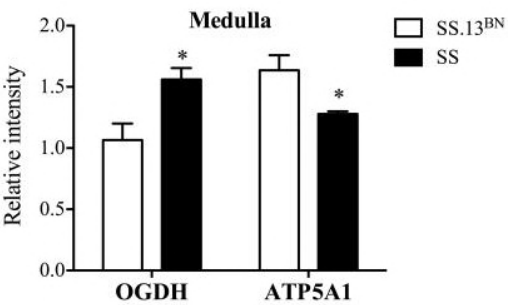

Figure 4

43
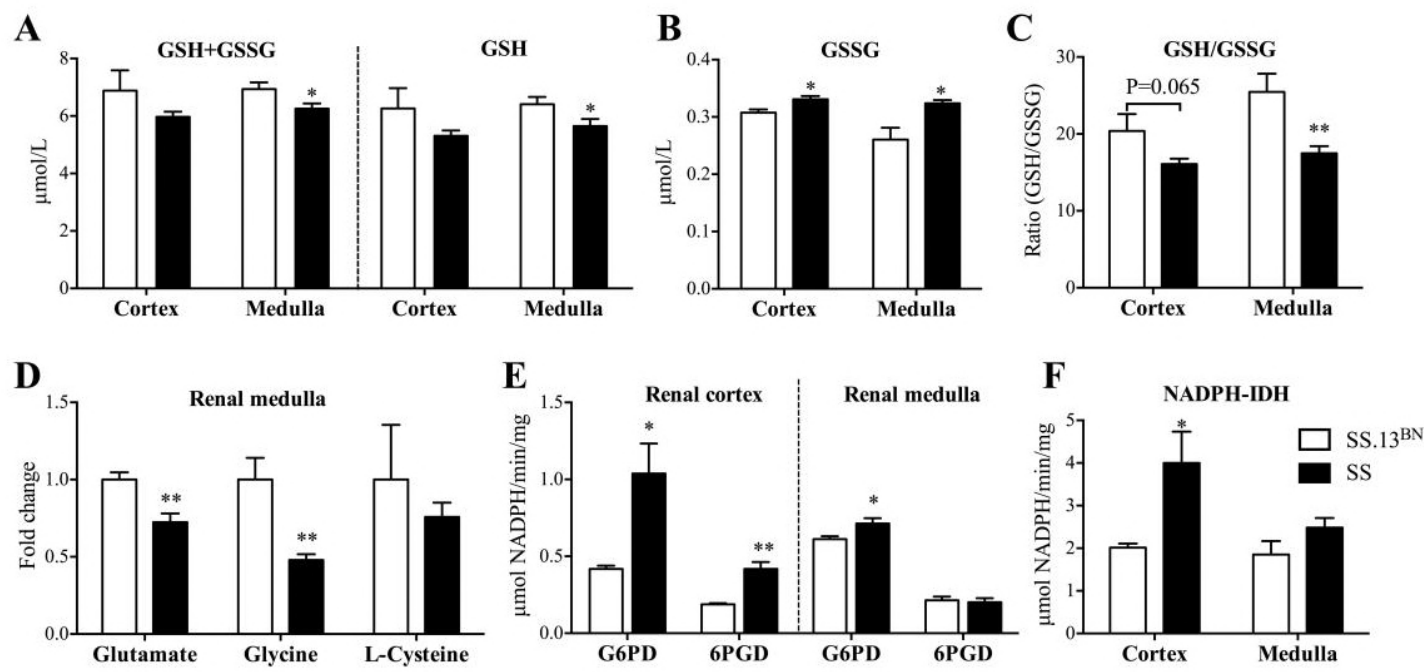
bioRxiv preprint doi: https://doi.org/10.1101/302182; this version posted April 16, 2018. The copyright holder for this preprint (which was not certified by peer review) is the author/funder. This article is a US Government work. It is not subject to copyright under 17 USC 105 and is also 54 made available for use under a CCO license.

55

56 Figure 5

A
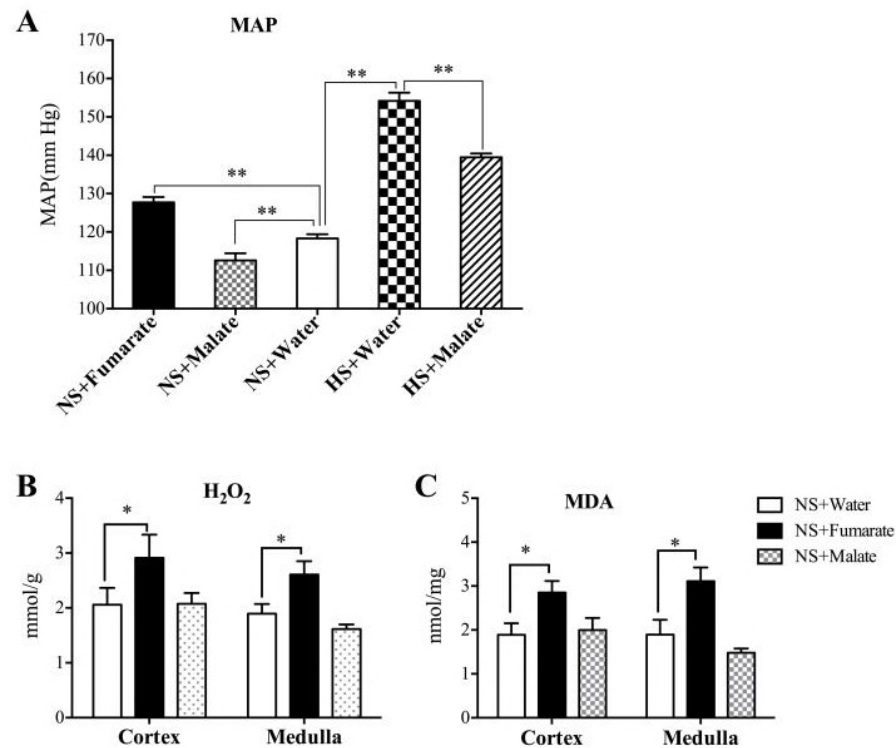

D

$\mathrm{H}_{2} \mathrm{O}_{2}$

$\mathbf{E}$
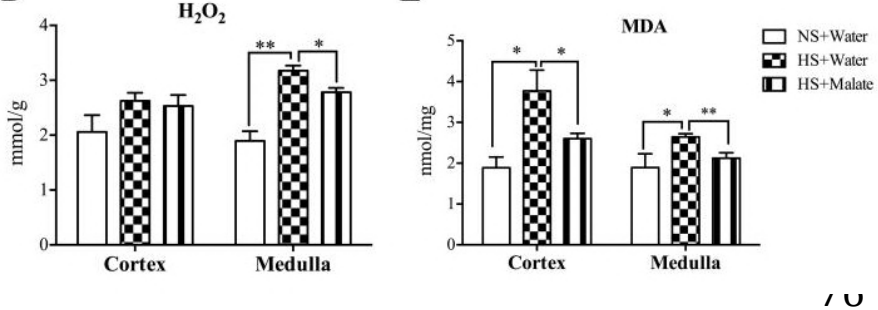